\title{
Common and Different Features Between Depression and Apathy in Neurocognitive Disorders
}

\section{Claudia lacobacci*}

Ph.D. Program in Behavioral Neuroscience, Sapienza University of Rome, Via dei Marsi 78, 00185 Rome, Italy Cognitive Neuroscience and Cerebellum Laboratory, Italy

Keywords: Depression; Apathy; Neurocognitive Disorders

\section{Introduction}

Depression and apathy are common neuropsychiatric disorders, they are often comorbid in different syndromes and share some signs. Both of these conditions present an overlap of core symptoms and this is the reason why is difficult to set apart them.

According to the Diagnostic and Statistical Manual of Mental Disorders (DSM-V) depression is considered a mental state of altered mood characterized by sadness, desperation, anhedonia and decreased interest in daily life activities, subjective sensation of discomfort, feelings of worthlessness and lack of self-esteem. Therefore apathy can be an aspect of depression in the manner in which there is a lack of motivation and a decreased interest in all or most daily activities, but apathy may be a discriminable clinical construct. In fact in person affected by depression the loss of interests is associated with feeling of sadness.

Apathy manifests with reduced interest and motivation but sadness and depressed mood are not present. The loss of motivation in apathy is considered primarily a difficulty to perform voluntary action and diminished goal-oriented behavior [1]. This condition, causes indifference and absence of interest in the environment and the failure to respond emotionally to external stimuli but there aren't sensations of discomfort and feeling of sadness. According to the DSM-V both depression and apathy cause clinically significant impairment in important areas of functioning.

Depression and apathy may be considered common symptoms in presence of neurological disease. In several neurodegenerative disorders such as frontotemporal dementia (FTD), Parkinson's disease, Alzheimer's disease (AD), subcortical dementia, apathy and depression may also represent the first symptoms of the disease and may precede the cognitive decline.

There are some differences in affective alterations between neurological and depressed subjects. In neurodegenerative diseases prominent symptoms may be apathy and irritably and patients don't show feeling of sadness, desperation or lack of self-esteem. Frequently in neurological disease, particularly in the early stages of the disease, in addition to behavioral changes and mood alterations may be present cognitive deficits, such as impairments in planning, inhibition and working memory.

Depression and apathy are both defined by poor initiative and loss of interest for daily life activities but these conditions can be differentiated according to cognitive and emotional features. Apathetic subjects are passive, they are not worry about their health condition and they are not complaining. They show flattened affect without emotional response to a situation that normally elicits emotion. Depressed patients feel uncomfortable and actively avoid social situations. They feel sad, hopeless, guilty, without self-esteem. They are pessimistic and they have many brushes with death and can committed suicide. Both apathetic and depressive subjects report sleep disorders, such as hypersomnia or sleeplessness, and alterations in weight. These symptoms are often included in a depressive disorder but they are present also in neurologic diseases. Therefor when we are in presence of a reduction of motivation, we must to distinguish between an "apathic syndrome" from the symptom "apathy" present in depression.

In dementia apathy represents the most common behavioral disturbance and the frequency of an apathic syndrome tend to increase with the severity of the disease. In fact in advanced state of dementia, a subjects cannot be responsive to the environmental stimulus and makes no spontaneous vocal sound. Some have proposed that apathy in neurodegenerative disorders lie on a continuum, from apathy on the milder end to akinetic mutism [2] considered as a state of limited responsiveness to the environment.

Despite depression and apathy can coexist in the same patient, some studies support the possibility to discriminate them from each other. In neurodegenerative disorders, depression can be explained also as a "reactions" to the diagnosis especially when the subject is aware of their disturbances. In $\mathrm{AD}$, apathy and depression are the most common behavioral symptoms and is difficult to differentiate them. Isolated apathy can appear frequently in moderate stage of the disease. Behavioral alterations and personality changes such as apathy, disinhibited behaviors, aggressiveness and agitation are the initial signs of a FTD. Subjects can feel without energy and motivation but can have preserved self-esteem. In depression a preserved self-esteem is extremely uncommon and frequently the subject feels guilty and appears to be depreciating with himself. Some clinicians report that apathy is more frequent than depression in dementia regardless of etiology [3].

Levy et al. [1] have found that the severity of apathy and depression can discriminate between $\mathrm{AD}$ and different forms of dementia. Patients affected by FTD and progressive supranuclear palsy (PSP) present more severe apathy and less severe depression than patients with $\mathrm{AD}$. In patients with mild cognitive impairment apathy might indicate increased probability to convert to $\mathrm{AD}$ a year later [4-6].

In dementia, apathy and depression may be due to a damage in certain cellular networks connecting different areas of the brain. In fact, brain dysfunction that cause neurologic symptoms also affects

${ }^{*}$ Corresponding author: Ph.D. Program in Behavioral Neuroscience, Sapienza University of Rome, Via dei Marsi 78, 00185 Rome, Italy, lacobacci C, Cognitive Neuroscience and Cerebellum Laboratory, Italy, Tel: + 39 06515011; E-mail: cla. iacobacci@gmail.com

Received: August 21, 2017; Accepted: August 29, 2017; Published: September 07, 2017

Citation: lacobacci C (2017) Common and Different Features Between Depression and Apathy in Neurocognitive Disorders. Clin Exp Psychol 3: 163. doi: 10.4172/2471-2701.1000163

Copyright: ( 2017 lacobacci C. This is an open-access article distributed under the terms of the Creative Commons Attribution License, which permits unrestricted use, distribution, and reproduction in any medium, provided the original author and source are credited. 
Citation: lacobacci C (2017) Common and Different Features Between Depression and Apathy in Neurocognitive Disorders. Clin Exp Psychol 3: 163. doi: 10.4172/2471-2701.1000163

Page 2 of 2

brain areas that regulate mood. The alterations involve these networks responsible for motivation, planning, judgment, and insight. These systems involve different subregions of the brain, principally the prefrontal cortex (PFC) and subcortical regions such as the basal ganglia. Apathy, in fact, is also a common feature in Parkinson's disease and Huntington's disease.

Also disorders involving neurochemical abnormalities in dopamine transmission, can cause a similar presentation. Dopamine, in fact, is principally involved in regulating pleasure, reward, motivation and goal-directed behavior.

In literature apathy has received less attention than depression, but is important distinguish these conditions from the viewpoint of its causes and treatment [7].

There aren't many medications or other treatments that have shown efficacy in treating apathy but assuming that apathy is associated with a decrease in dopaminergic transmission it has been hypothesized that dopamine-boosting treatments (such as Methylphenidate) may be effective in treating apathy. The project ADMET (Apathy in Dementia Methylphenidate Trial) is the first study in which have been identified and recruited $\mathrm{AD}$ patients with apathy and no major depressive episodes. This trial was designed to examine the efficacy and safety of Methylphenidate as treatment for clinically significant apathy in AD.

Results showed that Methylphenidate is effective in treating apathy in $\mathrm{AD}$. Changes in score of apathy evaluation scale are reported from baseline to 6 weeks and a further improvement was observed with respect to MMSE, suggesting that this treatment may be associated with an improvement in global cognition. These results support the concept that apathy can be categorized as a distinct behavioral syndrome in $\mathrm{AD}$ respect depression. Furthermore dopaminergic agonists seem to be effective for apathy but are not effective in depression. Apathy, instead, do not respond to antidepressive treatment, and indeed have been reported that antidepressive treatment in some cases increase the intensity of apathy [8]. Also, nonpharmacological interventions have relevant outcomes to treat apathy in dementia above all associated with pharmacological interventions. Nonpharmacological interventions include cognitive stimulation and creative activities, music therapy, pet therapy and doll therapy [9-15].

\section{Conclusion}

In conclusion, the relationship between apathy and depression appears complicated, but is important to differentiating these conditions. It is important because pharmacological approaches may be different and in some case drugs used to treat depression may worsen the symptoms of apathy. In every case the better choice consist to combined a pharmacological therapy and nonpharmacological intervention [16-20].

\section{References}

1. Levy ML, Cummings JL, Fairbanks LA, Masterman D, Miller BL, et al. (1998) Apathy is not depression. J Neuropsychiatry Clin Neurosci 10: 314-319.

2. Marin RS, Wilkosz PA (2005) Disorders of diminished motivation. J Head Trauma Rehabil 20: 377-88.

3. Chow TW, Binns MA, Cummings JL, Lam I, Black SE, et al. (2009) Apathy symptom profile and behavioral associations in frontotemporal dementia vs dementia of Alzheimer type. Arch Neurol 66: 888-893.

4. Robert PH, Berr C, Volteau M, Bertogliati C, Benoit M, et al. (2006) Apathy in patients with mild cognitive impairment and the risk of developing dementia of Alzheimer's disease: a one-year follow-up study. Clin Neurol Neurosurg 108: 733-736.

5. Robert PH, Berr C, Volteau M, Bertogliati C, Benoit M, et al. (2006) Neuropsychological performance in mild cognitive impairment with and without apathy. Dement Geriatr Cogn Disord 21: 192-197.

6. Robert PH1, Darcourt G, Koulibaly MP, Clairet S, Benoit M, et al. (2006) Lack of initiative and interest in Alzheimer's disease: a single photon emission computed tomography study. Eur J Neurol 13: 729-735.

7. Ishizaki J, Mimura M (2011) Dysthymia and apathy: Diagnosis and treatment. Depression Research and Treatment.

8. Barnhart WJ, Makela EH, Latocha MJ (2004) SSRI-induced apathy syndrome: A clinical review. J Psychiatr Pract 10: 196-9.

9. American Psychiatric Association (2013) Diagnostic and statistical manual of mental disorders.

10. Baquero M, Martín N (2015) Depressive symptoms in neurodegenerative diseases. World J Clin Cases 3: 682-693.

11. Brodaty H, Kim Burns K (2012) Nonpharmacological management of apathy in dementia: A systematic review.

12. Grossi D, Trojano L (2005) Neuropsychology of frontal lobes. Dissective syndromes and behavioral disorders.

13. Litvan I, Cummings JL, Mega M (1998) Neuropsychiatric features of corticobasal degeneration. J Neurol Neurosurg Psychiatry 65: 717-21.

14. Marin RS, Firinciogullari S, Biedrzycki RC (1994) Group differences in the relationship between apathy and depression. J Nerv Ment Dis 182: 235-239.

15. Marin RS, Firinciogullari S, Biedrzycki RC (1993) The sources of convergence between measures of apathy and depression. J Affect Disord 28: 7-14.

16. Marin RS (1991) Apathy: A neuropsychiatric syndrome. J Neuropsychiatry Clin Neurosci 3: 243-254

17. Marin RS (1996) Apathy: Concept, syndrome, neural mechanisms, and treatment. Semin Clin Neuropsychiatry 1: 304-314.

18. Marin RS (1990) Differential diagnosis and classification of apathy. Am J Psychiatry 147: 22-30.

19. Salamone JD, Correa M (2012) The mysterious motivational functions of mesolimbic dopamine. Neuron 76: 470.

20. Starkstein SE, Ingram L, Garau ML, Mizrahi R (2005) On the overlap between apathy and depression in dementia. J Neurol Neurosurg Psychiatry 76: 10701074. 